\title{
Qualidade de vida relacionada à saúde entre enfermeiros docentes
}

\author{
Quality of life related to health among teaching nurses \\ Calidad de vida relacionada con la salud en las enfermeras docentes
}

Recebido: 08/04/2021 | Revisado: 15/04/2021 | Aceito: 18/04/2021 | Publicado: 02/05/2021

\author{
Roseany Patrícia Silva Rocha \\ ORCID: https://orcid.org/0000-0002-2295-5321 \\ Universidade do Estado de Mato Grosso, Brasil \\ E-mail: roseanyrocha1@gmail.com \\ Thamiris dos Santos Bini \\ ORCID: https://orcid.org/0000-0001-6185-3902 \\ Universidade do Estado de Mato Grosso, Brasil \\ E-mail: thamirissantosbini@outlook.com \\ Antonia Dinágila do Nascimento Ribeiro \\ ORCID: https://orcid.org/0000-0001-7852-9199 \\ Universidade do Estado de Mato Grosso, Brasil \\ E-mail: dihribeiro1995@gmail.com \\ Grasiela Cristina Silva Botelho Silvestre \\ ORCID: https://orcid.org/0000-0001-5367-4648 \\ Universidade do Estado de Mato Grosso, Brasil \\ E-mail: enf.grasielabotelho@gmail.com \\ Renata Tomazelli Ferreira \\ ORCID: https://orcid.org/0000-0002-7283-478X \\ Universidade do Estado de Mato Grosso, Brasil \\ E-mail: renatatomferreira@gmail.com
}

\begin{abstract}
Resumo
Objetivo: Está pesquisa teve o intuito de avaliar a qualidade de vida relacionado a saúde (QVRS) de enfermeiros docentes e preceptores em uma Universidade do Estado de Mato Grosso. Método: Trata-se de um estudo descritivo de corte transversal, com abordagem quantitativa. Foram aplicados dois instrumentos, sendo o primeiro que avaliou o perfil sócio demográfico, profissional e de saúde, e o segundo avaliou a qualidade de vida (QVRS), em agosto de 2020. Resultados: Dos 27 docentes ouve a prevalência foi do sexo feminino, 20 (74\%), seguido da faixa etária 31 anos $15(55,6 \%)$ e $7(26,0 \%)$ tinham doença crônica sendo a hipertensão arterial a mais prevalente. Ao avaliar a distribuição dos domínios de qualidade de vida foi evidenciado escores alto para capacidade física $(82,48)$, seguido de saúde mental $(59,85)$. Os docentes vêm sofrendo com as mudanças sociais, econômicas, tecnológicas nesse cenário atual de pandemia que estamos enfrentando, o que está dando novos rumos e sentido ao trabalho, ocasionando desgastes físicos, psicológicos e comportamentais, o que pode acarretar de forma negativa a sua saúde mental. Conclusão: Assim, conclui-se importância e relevância do tema para a gestão do trabalho em enfermagem na docência, sugere-se novos estudos considerando a amplitude de possibilidades que abarcam este assunto, sem contar na intencionalidade de provocar a preparação, reflexão, compreensão e planejamento para que docentes tenham uma melhor qualidade de vida.
\end{abstract}

Palavras-chave: Qualidade de vida; Trabalho; Docente de enfermagem.

\begin{abstract}
Objective: This research aimed to assess the health-related quality of life (HRQoL) of nurse professors and preceptors at a University of the State of Mato Grosso. Method: This is a descriptive cross-sectional study, with a quantitative approach. Two instruments were applied, the first of which assessed the socio-demographic, professional and health profile, and the second assessed the quality of life (HRQoL), in August 2020. Results: Of the 27 teachers who heard the prevalence, it was female, 20 (74\%), followed by the age group 31 years old $15(55.6 \%)$ and $7(26.0 \%)$ had chronic disease, with arterial hypertension being the most prevalent. When assessing the distribution of quality of life domains, high scores for physical capacity (82.48) were evidenced, followed by mental health (59.85). Teachers have been suffering from social, economic, technological changes in this current pandemic scenario that we are facing, which is giving new directions and meaning to work, causing physical, psychological and behavioral wear, which can negatively affect your health mental. Conclusion: Thus, it concludes the importance and relevance of the theme for the management of nursing work in teaching, further studies are suggested considering the breadth of possibilities that
\end{abstract}


cover this subject, not to mention the intentionality of provoking preparation, reflection, understanding and planning for teachers to have a better quality of life.

Keywords: Quality of life; Work; Faculty nursing.

\begin{abstract}
Resumen
Objetivo: Esta investigación tuvo como objetivo evaluar la calidad de vida relacionada con la salud (CVRS) de profesores y preceptores de enfermería de una Universidad del Estado de Mato Grosso. Método: Se trata de un estudio descriptivo transversal, con enfoque cuantitativo. Se aplicaron dos instrumentos, el primero de los cuales evaluó el perfil sociodemográfico, profesional y de salud, y el segundo evaluó la calidad de vida (CVRS), en agosto de 2020. Resultados: De los 27 docentes que escucharon la prevalencia, fue del sexo femenino. 20 (74\%), seguido del grupo de edad de 31 años $15(55,6 \%)$ y $7(26,0 \%)$ tenían enfermedad crónica, siendo la hipertensión arterial la más prevalente. Al evaluar la distribución de los dominios de la calidad de vida, se evidenciaron puntuaciones altas para la capacidad física $(82,48)$, seguidas de la salud mental $(59,85)$. Los docentes vienen sufriendo los cambios sociales, económicos, tecnológicos en este actual escenario pandémico al que nos enfrentamos, que está dando nuevos rumbos y sentido al trabajo, provocando un desgaste físico, psicológico y conductual, que puede afectar negativamente su salud mental. Conclusión: Así, se concluye la importancia y relevancia del tema para la gestión del trabajo de enfermería en la docencia, se sugieren estudios adicionales considerando la amplitud de posibilidades que cubre este tema, sin mencionar la intencionalidad de provocar preparación, reflexión, comprensión y planificación para que los profesores tengan una mejor calidad de vida.
\end{abstract}

Palabras clave: Calidad de vida; Trabajo; Docentes de enfermería.

\title{
1. Introdução
}

O trabalho é um ato importante na vida social e histórica dos indivíduos, além de ser um meio de construção de identidade pessoal. Durante sua prática profissional o enfermeiro enfrenta diferentes desafios que se relacionam com o modo de organização do trabalho na produção do serviço de saúde, são elas a forma de inserção da enfermagem no mundo do trabalho, multiemprego, jornadas extensas, sobrecarga de trabalho, remuneração abaixo da expectativa, baixo reconhecimento na equipe de saúde, dor e sofrimento ao cuidar de pacientes e familiares, jornada noturna (Miorin, 2018).

A qualidade de vida no trabalho é caracterizada pelo bem-estar do indivíduo, proporcionando satisfação e segurança no ambiente laboral, tendo para si o apoio da instituição em que está inserido, assim estará alcançando toda a expectativa de vida e realizando os objetivos desejados (Zavala, Klinj \&Carrillo, 2016).

O enfermeiro docente desenvolve o seu trabalho de uma forma ampla, no exercício sistemático de acompanhamento e orientação profissional na educação e estágios curriculares, tendo como sua principal função o de mediador do processo de ensino e aprendizagem construído na pratica. Portanto o enfermeiro, além de possuir suas responsabilidades de ensino, colabora na organização, execução e avaliação da instituição, nos estágios supervisionados e práticas de campo que são desenvolvidos nas unidades de saúde, tendo o intuito de supervisionar, orientar e participar de todos os desempenhos do acadêmico (Carvalho \& Fagundes, 2008).

A QVRS no trabalho, tem sido um dos principais motivos para a pesquisa científica. Para desenvolver suas atividades de ensino, o enfermeiro enfrenta diversos desafios, como, a desvalorização salarial, escassez de material didático disponível, rotina cansativa e por vezes uma estrutura física inadequada. Essas características, é proveniente da própria organização do trabalho, mas que podem comprometer a sua saúde e Qualidade de Vida (QV), pois o enfermeiro se vê na necessidade de ter mais um vínculo empregatício, interferindo assim, na sua saúde física e mental ao longo da atividade laboral (Caveião et al., 2017).

Com o advento da pandemia, a educação foi um dos seguimentos sociais que necessitou interromper as atividades pedagógicas. Isso deu-se a partir de portarias instituídas pelo Ministério da Educação, que determinou aos gestores de instituições de nível superior e seus docentes a necessidade de adaptar e elaborar estratégias de operacionalização das atividades pedagógicas de forma remota durante esse momento de pandemia (Silva; Estrela; Lima \& Abreu, 2020). 
Diante dessa necessidade, os docentes ficaram expostos a novas realidades de ensino- aprendizagem que impuseram inúmeros desafios a serem superados mesmo diante do medo e incertezas sobre a doença. Nesse contexto, esses profissionais estão vulneráveis a ansiedade, pressões das instituições de ensino, sobrecarga de trabalho emocional e estresse, o que pode reverberar no adoecimento, principalmente relacionado à saúde mental (Silva; Estrela; Lima; Abreu, 2020).

Portanto, justifica-se a importância desse estudo, pois possibilitará, uma melhor compreensão da qualidade de vidaQVRS dos enfermeiros docentes e quais estão sendo os fatores que estão influenciando na QVRS, e esse tem grande potencial de contribuir com o avanço nos cuidados a saúde do trabalhador.

Entretanto, enfatiza-se a necessidade de exploração ainda de forma mais ampliada da identificação da Qualidade de Vida do enfermeiro que atua no ensino superior. Essa pesquisa ancora-se na seguinte questão norteadora: Qual é a QVRS relacionada a saúde do enfermeiro docente? Tendo como objetivo avaliar a qualidade de vida relacionado a saúde (QVRS) relacionado à saúde dos enfermeiros docentes de uma universidade no Estado de Mato Grosso.

\section{Metodologia}

Trata-se de um estudo descritivo de corte transversal, com abordagem quantitativa, realizado em uma instituição pública de ensino superior, localizada no interior do Estado de Mato Grosso. A pesquisa quantitativa está relacionada com os pensamentos positivista lógicos, com enfoque ao raciocínio dedutivo, regras lógicas onde as informações são traduzidas em opiniões através dos números e com os atributos mensuráveis da experiência humana. A pesquisa quantitativa é um método que tem como objetivo utilizar os procedimentos metodológicos onde tudo pode ser quantificável, o qual significa traduzir os números em opiniões e informações para classificá-las e analisá-las. Esta abordagem busca quantificar as opiniões e informações dos resultados obtidos da pesquisa, por meio do uso de recursos e de técnicas estatísticas de porcentagem, média, moda, mediana, desvio padrão, e outros (Gil, 2010; Polit, Beck, 2011).

A população de estudo foi constituída pelos enfermeiros que atuavam como docentes na referida instituição. No período da coleta de dados, a instituição possuía o total 31 enfermeiros docentes, destes, 4 não responderam o instrumento, contabilizados o total de 27 participantes incluídos na pesquisa.

Os critérios de inclusão estabelecidos foram: ser graduado em enfermagem e atuar como docente na referida universidade. Como critério de exclusão, foi considerado a condição de afastamento do trabalho por motivo de doença, licença maternidade ou férias no período da coleta de dados.

A coleta dos dados ocorreu no mês de agosto de 2020. Dado o contexto da pandemia e a impossibilidade de a coleta ser realizado pessoalmente, dois instrumentos foram enviados no e-mail institucional dos docentes via plataforma Google forms, sendo o primeiro que identificou o perfil sociodemográfico, histórico profissional, e hábitos de saúde; e o segundo que foi o The Medical Outcomes Study 36-item Short-Form Health Survey (SF-36) para mensurar a qualidade de vida relacionado a saúde. Incialmente o docente assinou o Termo de Consentimento Livre e Esclarecido - TCLE, e em seguida respondeu os instrumentos.

Os dados foram codificados e duplamente digitados e compuseram um banco de dados com recursos do Programa Epidata versão 7.2. Após a coleta, os dados foram sistematizados em planilhas eletrônicas e posteriormente o banco de dados foi conferido por dois pesquisadores de forma independente. Após a digitação, os dados foram verificados quanto a possíveis erros e/ou inconsistência. Considerando o desenho do estudo, as variáveis foram descritas por sua distribuição, frequência absoluta e relativa e valores médios.

Destaca-se que, o presente estudo não fere os princípios éticos que tratam dos estudos envolvendo seres humanos, pois o projeto de pesquisa foi submetido e aprovado pelo Comitê de Ética em Pesquisa da Unemat sob o $n^{\circ}$ do CAAE: 
29329020.3.0000.5166 e parecer de aprovação $\mathrm{n}^{\circ}$ 4.183.872, em atendimento as diretrizes da Resolução 466/2012, do Conselho Nacional de Saúde, e suas complementariedades.

\section{Resultados}

As informações utilizadas para o presente estudo foram organizadas por meio da análise dos 27 questionários, respondidos pelos enfermeiros docentes, seguindo os critérios de inclusão adotados.

Na caracterização quanto ao sexo, conforme demonstrado a (Tabela 1), obteve-se a predominância do sexo feminino de 20 (74\%). Em relação à idade destaca-se a faixa etária acima de 31 anos, com 15 (55,6\%). No que se refere ao estado civil, $15(55,6 \%)$ declararam ser casados, $11(40,7 \%)$ vivem sem a presença do companheiro (solteiros, separados e viúvos), 15 $(55,6 \%)$ declararam ter filhos, sendo que destes $8(29,6 \%)$ afirmaram ter apenas um filho. Verificou-se que o maior percentual de enfermeiros, 12 (44,4\%), afirmaram ser o (a) esposo (a) o chefe da família e 11 (40, 7\%) dos profissionais se autodeclararam chefes de família.

Tabela 1. Distribuição das características relacionadas ao perfil sócio demográfico dos enfermeiros docentes. Diamantino (MT). 2020.

\begin{tabular}{cccc}
\hline VARIÁVEIS & & $\boldsymbol{n}$ & $\boldsymbol{\%}$ \\
\hline \multirow{2}{*}{ Sexo } & Feminino & 20 & 74,0 \\
& Masculino & 7 & 26,0 \\
Faixa etária & 24 a 30 anos & 12 & 44,4 \\
& Mais de 31 anos & 15 & 55,6 \\
& & & \\
& Solteiro (a) & 11 & 40,7 \\
Estado Civil & Casado (a) & 15 & 55,6 \\
& Viúvo (a) & 1 & 3,7 \\
Possui filhos & Sim & 15 & 55,6 \\
& Não & 12 & 44,4 \\
Quantos? & 1 filho & 8 & 53,3 \\
& 2 filhos & 7 & 46,7 \\
& & 12 & 44,4 \\
Quem é chefe da família? & Esposo(a)/Companheiro(a) & 4 & 14,9 \\
& Pai/mãe & 11 & 40,7 \\
\hline
\end{tabular}

Fonte: Autores.

Quanto a natureza da atuação profissional, observou-se que o tempo de trabalho na instituição foi entre 1 a 2 anos, 10 (37\%). Quanto a maior qualificação profissional, 13 (48,1\%) afirmaram ser mestres e ou doutores. E da população total 20 (74\%) não possuem segundo vínculo empregatício. 
Tabela 2. Distribuição das características relacionadas à formação profissional dos enfermeiros docentes. Diamantino (MT). 2020 .

\begin{tabular}{|c|c|c|c|}
\hline VARIÁVEIS & & $n$ & $\%$ \\
\hline \multirow{6}{*}{$\begin{array}{c}\text { Tempo de Trabalho na } \\
\text { instituição }\end{array}$} & & 10 & 37,0 \\
\hline & 1 a 2 anos & 3 & 11,1 \\
\hline & 3 a 4 anos & 7 & 26,0 \\
\hline & 5 a 6 anos & 3 & 11,1 \\
\hline & 7 a 8 anos & 4 & 14,9 \\
\hline & Não respondeu ao tempo de trabalho & & \\
\hline \multirow[t]{3}{*}{$\begin{array}{c}\text { Maior Qualificação na área } \\
\text { da saúde }\end{array}$} & Graduação & 4 & 14,9 \\
\hline & Latu sensu (Especialização) & 10 & 37,0 \\
\hline & Stricto sensu (Mestrado e/ou Doutorado) & 13 & 48,1 \\
\hline \multirow{2}{*}{ Possui segundo vinculo } & Sim & 7 & 26,0 \\
\hline & Não & 20 & 74,0 \\
\hline
\end{tabular}

Fonte: Autores.

No que diz respeito ao perfil sobre hábitos de vida e saúde dos enfermeiros docentes, os resultados que contribuíram para a construção do perfil revelaram que 14 (51,9\%) não fazem uso de bebida alcoólica e que 27 (100\%) afirmaram não serem tabagistas.

Ainda, observou-se que entre os entrevistados 7 (26\%) declararam possuir doença (s) crônica (s) diagnosticadas e fazerem uso permanente de medicamentos. Entre as doenças declaradas, se destacaram a Hipertensão Arterial Sistêmica (HAS), renite e carências de vitaminas. Sobre o histórico de uso frequente de medicações, 10 (37\%) declaram fazer uso de medicações de forma contínua. 
Tabela 3. Distribuição das características relacionadas aos hábitos e o perfil à saúde dos enfermeiros docentes. Diamantino (MT). 2020.

\begin{tabular}{cccc}
\hline VARIÁVEIS & Sim & \% & 48,1 \\
\hline Faz uso de bebida & Não & 13 & 51,9 \\
alcoólica? & Sim & 14 & 100 \\
É tabagista? & Não & 0 & 27 \\
Tem diagnóstico de alguma & Sim & 26,0 & 74,0 \\
doença crônica? & Não & 7 & 3,7 \\
& & 20 & 3,7 \\
Quais? & Carência de vitamina D. & 1 & 7,4 \\
& Rinite crônica. & 1 & 3,7 \\
Faz uso regular de & Hipertensão Arterial. & 2 & 3,7 \\
medicamento? & Sinusite. & 1 & 3,7
\end{tabular}

Fonte: Autores.

A Tabela 4 apresenta as médias, medianas e desvio padrão dos escores dos domínios de QVRS (SF-36). O escore médio da QVRS foi de $(56,34)$ e o domínio com a menor média foi estado geral de saúde $(34,56)$, e com a maior média foi capacidade física $(82,48)$.

Tabela 5. Distribuição dos domínios, relacionada a qualidade de vida dos enfermeiros docentes. Diamantino (MT). 2020.

\begin{tabular}{cccc}
\hline Domínios & Média & Mediana & Desvio padrão \\
\hline Capacidade Física & 82,48 & 85 & 16,08 \\
$\begin{array}{c}\text { Limitação por aspectos } \\
\text { físicos }\end{array}$ & 55,55 & 50 & 39,42 \\
Dor & 58,55 & 52 & 26,02 \\
Estado geral de saúde & 56,03 & 57 & 14,38 \\
Vitalidade & 50,74 & 50 & 18,48 \\
Aspectos sociais & 50 & 50 & 27,07 \\
Limitação por aspectos & & & 38,65 \\
emocionais & 34,56 & 33,33 & 19,29 \\
Saúde Mental \\
QVRS
\end{tabular}

Fonte: Autores (2020). 


\section{Discussão}

O gênero feminino foi predominante, demostrando que a feminilização na área da enfermagem tem permanecido por décadas devido razão tradicional e cultural e a crescente representação da mulher no mercado de trabalho e na sociedade (Machado, 2016; Scholze, 2016).

A faixa etária está acima de 31 anos com o porcentual de 15 (55,6\%). Estudos abordam em relação a idade o fato que a enfermagem é uma profissão em pleno rejuvenescimento, registrando-se: $40 \%$ do seu contingente nacional com idade entre 36-50 anos; (38\%) (Machado, 2016).

Em relação ao estado civil, identificou-se a prevalência de indivíduos casados. Tais resultados vão de encontro com um determinado estudo que buscou caracterizar o perfil da enfermagem brasileira, e constatou-se que nacionalmente dos 414.712 enfermeiros participantes, 50,9\% declararam possuir companheiro e tendo filhos.

Com relação ao tempo na docência, podemos perceber que a maior prevalência foi entre 1 a 2 anos, o que corrobora com a literatura cientifica (Franco; Fernandes; Millão, 2020), em que a experiência dos enfermeiros docentes são de no máximo cinco anos. O tempo e a experiência no ambiente de trabalho são essenciais para enfermeiro docente e vale destacar que o trabalho é algo fundamental na compreensão da história do homem, entende-se que o homem, enquanto construtor da sua própria história, produz a sua própria existência através do trabalho.

Em relação à formação profissional, destacou-se a qualificação em nível stricto sensu, mestrado e/ou doutorado. Tais dados expressam que o campo de trabalho tem exigido cada vez mais uma qualificação dos profissionais da saúde em prol da ascensão da carreira, que posteriormente pode proporcionar uma maior satisfação e maior remuneração. Destaca-se ainda que a qualificação proporciona uma melhor qualidade de ensino aos estudantes (Silva; Estrela; Lima; Abreu, 2020).

Em relação ao segundo vínculo mais da metade dos docentes não possui mais de um vínculo empregatício o que é positivo para a saúde, considerando que exercer longas jornadas de trabalho é um fator de risco para acidentes de trabalho, doenças crônicas como hipertensão arterial (HA), problemas muscoesqueléticos, e estresse ocupacional. Estes dados também têm relação com o fato de ser uma instituição pública, com um contrato de trabalho reduzido e acessível e que requer dedicação exclusiva e mais esforço na produção de conhecimento para os estudantes de enfermagem (Bublitz et al., 2019).

Os hábitos de vida e saúde indicaram que a maior parte consome bebidas alcoólicas moderadamente e todos não são tabagistas. Os resultados corroboram com um determinado estudo (Domingues et al., 2019) onde a maioria dos enfermeiros docentes declararam não ser fumante $(90,8 \%)$ e não consumir bebida alcoólica $(55,1 \%)$. A possível justificativa para esses dados seria o fato de que enquanto enfermeiros esses conhecem os malefícios causados e, por conseguinte, evitam o consumo dessas substâncias.

Em relação as doenças ocupacionais, pode se dá pela falta de conhecimento sobre as medidas preventivas no trabalho, e todo o conjunto de desordem que possa afetar a saúde do trabalhador.

Além disso, apenas 7 (26\%) dos docentes relataram possuir Doenças Crônicas não Transmissíveis (DCNT), estas podem se desenvolver por fatores multicausais. Ao levar este assunto para o campo da docência, percebe-se que os efeitos deletérios deste serviço podem influenciar na saúde do trabalhador, visto que as condições psicossociais são fatores que podem vir a contribuir para o aumento da vulnerabilidade e dos riscos de DCNT (Silva; Estrela; Lima; Abreu, 2020).

Constatou-se também que alguns docentes fazem o uso de medicação contínua, a necessidade do uso de medicação acontece devido as multimorbidades influenciadas por diversos fatores, consequentemente pode levar à queda da qualidade de vida (Melo et al., 2019; Pimenta et al., 2016).

Sobre os dados da QVRS, identificou-se escore médio de 56,34, menor média no domínio estado geral de saúde $(34,56)$, e maior média para os aspectos sociais $(82,48)$. Neste sentido, os resultados corroboram com um estudo que avaliou a 
QVRS dos profissionais de enfermagem, variando a média de 80 a 100 que é considerada condições razoáveis de saúde e qualidade de vida (Costa et al., 2017).

Aos aspectos sociais, os dados apresentados neste estudo são semelhantes a pesquisa que compararam a QV dos diversos profissionais (Médicos, Fisioterapeutas, Enfermeiros e Técnicos de Enfermagem), independente da sua jornada de trabalho e do seu local de residência, o autor relata que indivíduos que praticavam atividade física apresentaram maior escores neste domínio (Costa et al., 2017).

A Limitação por aspectos físicos possui uma média de $(55,55)$ o que é considerada baixa. Esse resultado nos revela que os enfermeiros docentes possuem algumas limitações para concluir atividades que gera esforços físicos. Podemos relacionar esses dados ao fato que a uma prevalência do sexo feminino na profissão, e além das atividades referente ao trabalho, as mulheres exercem outros afazeres como os serviços domiciliares, maternidade o que somado as atividades do trabalho pode leva-las ao estresse ocupacional, físico e mental (Albuquerque; Silva; Oliveira, 2018).

O domínio dor apresentou-se significativamente na população em estudo. Corroborando a este estudo, uma pesquisa realizada com 106 profissionais enfermeiros, buscou analisar a qualidade de vida no trabalho em uma maternidade escola, os autores identificaram que os indivíduos que possuíam dupla jornada em setor de assistência direta, a dor foi o domínio que mais se apresentou prejudicado, onde 27,3\% dos indivíduos apresentaram escore menor que 50 (Albuquerque; Silva; Oliveira, 2018).

O domínio de vitalidade, mostra prejuízo no vigor físico e energia, em que correlaciona com as dificuldades relacionadas aos aspectos sociais, referente as atividades, como ter tempo de socializar-se, tempo este em que as relações de trabalho afetam as dimensões física e emocional, o domínio de saúde mental está relacionado ao quanto este profissional sentese angustiado, deprimido ou infeliz em relação ao seu estado de saúde, e no domínio de avaliação da dor, avalia-se sua intensidade, bem como sua extensão ou sua interferência nas atividades de vida diária (Costa et al., 2017).

Prado (2016), enfatiza que os profissionais docentes sofrem impacto significativo com as mudanças sociais, econômicas, tecnológicas e principalmente no cenário atual de pandemia que estamos enfrentando, o que está dando novos rumos e sentido ao trabalho, ocasionando desgastes fisiológicos, cognitivos, físicos, psicológicos e comportamentais, o que pode acarretar de forma negativa a sua saúde mental.

Por fim, como em estudos verificados na literatura, percebeu-se um número reduzido de estudos que utilizaram o SF36 para avaliar a QVRS em enfermeiros docentes, o que implica em limitações na comparação dos achados. No entanto, também apontam para a importância de investigar esse fenômeno nessa população que possui características específicas de trabalho.

\section{Considerações Finais}

Conclui-se que maioria dos participantes deste estudo são do gênero feminino, com a idade superior a 31 anos, vivendo com a presença de companheiro e se encontram em exercício profissional. Não apresentaram a situação de multiemprego, trabalhando apenas na instituição de ensino em estudo. Alguns docentes relataram doenças crônicas, além de observar um pequeno grupo que fazem uso de medicação contínua.

O estudo alcançou o objetivo desta pesquisa que foi avaliar a qualidade de vida relacionado a saúde- QVRS dos enfermeiros docentes.

Ao mensurar a QVRS, notou-se média acima dos 55 pontos, sendo menor no domínio Limitação por aspectos emocionais e maior na capacidade física.

Como limitação do estudo, percebeu-se um número reduzido de pesquisas que utilizaram o SF-36 para avaliar a QVRS entre enfermeiros docentes, o que implicou na comparação dos achados com outras experiências de pesquisa. 
A pesquisa buscou contribui para exploração sobre a temática proposta e, dada sua importância e relevância para a gestão do trabalho em enfermagem na docência, sugere-se novos estudos considerando a amplitude de possibilidades que abarcam este tema, sem contar na intencionalidade de provocar a preparação, reflexão, compreensão e planejamento para que docentes tenham uma melhor QV. Ademais, espera-se que esse estudo sirva como referência à reflexão aos movimentos sociais protagonizadas pelas entidades de classe da enfermagem brasileira, no sentido da luta por melhores condições de trabalho da categoria, a partir da compreensão da natureza e características que lhe são próprias.

\section{Referências}

Albuquerque, S. M. P, Silva, A. K. L, \& Oliveira, H. C. (2018). Qualidade de vida no trabalho em enfermeiros de uma maternidade escola. Rev. Eeletrônica psicologia, 1-14. https://www.psicologia.pt/artigos/textos/a1295.pdf

Bublitz, S., Beck, C. L. C., Silva, R. M., \& Sturbelle, I. C. S. (2019). Perfil dos enfermeiros docentes atuantes em programas de pós-graduação "stricto sensu” de instituições públicas. Rev. Enferm. UFSM - REUFSM, 9(5), 1-15

Carvalho, E. S. D. S., \& Fagundes, N. C. (2018). A inserção da preceptoria no curso de graduação de enfermagem. Rene.

Caveião, C. et al. (2017). Perfil e qualidade de vida de docentes enfermeiros de universidades privadas e pública. Rev. APS, 20(2), 185-193. https://doi.org/10.34019/1809-8363.2017.v20.15819

Conceição, M. R. D. et al. (2012). Qualidade de vida do enfermeiro no trabalho docente: estudo com o whoqol-bref. Rev. Esc Anna Nery, 16 (2), 320 - 325. http://dx.doi.org/10.1590/S1414-81452012000200016

Costa, K. N. F. M. Costa, T. F, Marques, D. R. F, Viana, L. R. C. et al. (2017). Qualidade de vida relacionada à saúde dos profissionais de enfermagem. Rev Enferm UFPE Line, 11(2), 881-889. 10.5205/reuol.10263-91568-1-RV.1102sup20170

Domingues, J. G. et al. (2019). Doenças crônicas não transmissíveis em profissionais de enfermagem de um hospital filantrópico no Sul do Brasil. Epidemiol. Serv. Saúde, 28(2), 1-10. http://dx.doi.org/10.5123/s1679-49742019000200011

Franco, M. T., Fernandes, M. T. C., \& Millão, L. F. (2020). Perfil de enfermeiros-professores da educação profissional técnica de nível médio em enfermagem. Saúde coletiva, 10(56), 3164-3169. https://doi.org/10.36489/saudecoletiva.2020v10i56p3164-3175

GIL, Antônio Carlos. (2010). Como elaborar projetos de pesquisa. Atlas.

Machado, M. H. et al. (2016). Características gerais da enfermagem: o perfil sócio demográfico. Enferm. Foco, 7(6), 09-14. https://doi.org/10.21675/2357707X.2016.v7.nESP.686

Melo, L. A., Braga, L. C, Leite, F. P. P., Bittar, B. F., et al. (2019). Fatores associados à multimorbidade em idosos: uma revisão integrativa da literatura. Rev. bras. Geriatria gerontologia, 22(1), 1-11. https://doi.org/10.1590/1981-22562019022.180154

Morin, J. D. et al. (2018). Prazer e sofrimento de trabalhadores de enfermagem de um pronto-socorro. Texto contexto em enfermagem. 27(2), 2-9. https://doi.org/10.1590/0104-070720180002350015

Pimenta, A. M., \& Assunção, A. Á. (2016). Estresse no trabalho e hipertensão arterial em profissionais de enfermagem da rede municipal de saúde de Belo Horizonte, Minas Gerais, Brasil. Rev Bras Saúde Ocupacional, 41(6), 1-11. https://doi.org/10.1590/2317-6369000113515

Polit, D. F, \& Beck, C. T. (2011). Fundamentos de pesquisa em enfermagem:métodos, avaliação e utilização. Artmed.

Prado, C. E. P. (2016). Estresse ocupacional: Causas e consequências. Rev. Bras Med Trab, 14(3), 285-289. 10.5327/Z1679-443520163515

Scholze, A. R. (2016). Ambiente de trabalho, consumo de substâncias psicoativas e estresse entre enfermeiros hospitalares: análise dos fatores associados. (Dissertação). Londrina, 73 f.

Silva, A. F., Estrela, F. M., Lima, N. S., \& Abreu, C. T. A. (2020). Saúde mental de docentes universitários em tempos de pandemia. Physis: Revista de Saúde Coletiva, 30(2), 1-4. http://dx.doi.org/10.1590/S0103-73312020300216

Souza, R. F. et al. (2018). Repercussões dos fatores associados à qualidade de vida em enfermeiras de unidades de terapia intensiva. Rev. Salud Pública, 20(4), 453-459. https://doi.org/10.15446/rsap.V20n4.65342

Zavala, M. O. Q., Klinj, T. P., \& Carrillo, K. L.S. (2016). Qualidade de vida no trabalho do pessoal de enfermagem de instituições públicas de saúde. Rev. Latino-Am, 24(2713), 2-8. 10.1590/1518-8345.1149.2713 